\title{
Subcutaneous implantable cardioverter defibrillator device malfunction: first report of a "high current" condition triggering device failure
}

\author{
Kelvin C. Lau • Maully J. Shah
}

Received: 31 December 2014 / Accepted: 4 February 2015 /Published online: 20 February 2015

(C) Springer Science+Business Media New York 2015

\section{Case report}

A 16-year-old asymptomatic male with idiopathic hypertrophic cardiomyopathy underwent implantation of an implantable cardioverter defibrillator (ICD) for primary prevention of sudden cardiac death after he progressed to massive left ventricular hypertrophy (septal thickness $35 \mathrm{~mm}, Z=+13.72$ ). A Cameron Health/Boston Scientific (St. Paul, MN) SQ-RX Model 1010 subcutaneous ICD (S-ICD) and Model 3010 electrode were implanted uneventfully. The patient's 2-week and 6-month postimplantation S-ICD evaluations revealed no arrhythmia episodes and satisfactory electrode impedance status and battery longevity. At his 1-year follow-up visit, his S-ICD could not be interrogated even after changing of programmers and magnet reset. Most notably, there were no audible tones with magnet application. Premature battery depletion was suspected, although a review confirmed that his device did not fall within Cameron Health's field safety advisory in 2011 regarding a specific subset of SQ-RX Model 1010 generators that were prone to this problem. The manufacturer's safety advisory in 2013 concerning a necessary device software update to prevent the unintentional activation of an internal protective fuse also did not apply. Device explantation was therefore performed.

K. C. Lau $(\bowtie) \cdot$ M. J. Shah

Division of Cardiology, Department of Pediatrics, The Children's

Hospital of Philadelphia, Perelman School of Medicine at the

University of Pennsylvania, 34th Street and Civic Center Boulevard,

Philadelphia, PA 19104, USA

e-mail: lauk@email.chop.edu
Manufacturer's analysis of the explanted device revealed the activation of the battery fuse. Microscopic inspection found several damaged solder connections, resulting in small "flakes" that likely became dislodged and came into contact with the battery terminals to create a brief high current state. This activated the battery fuse, which serves to shield the patient from exposure to potentially high thermal conditions caused by a sustained short circuit. This, in turn, rendered the S-ICD inoperable.

\section{Discussion}

This is the first report of a S-ICD failure secondary to a high current condition (Manufacturer and User Facility Device Experience report \#MW5038415). For our patient, device failure was detected only upon routine follow-up because audible alarms became disabled with triggering of the battery fuse. This particular ICD technology also lacks remote monitoring, which could have provided an earlier notification of device inoperability. The duration of time in which our patient was "unprotected" may thus have been as long as the 6-month time interval between his in-office evaluations. Close surveillance of S-ICD recipients should therefore be maintained due to the unpredictable nature of this problem.

Conflict of interest Maully Shah has received an educational grant from Medtronic, Inc. The remaining author has no conflicts of interest. 